\title{
INDIVIDUAL BEE'S EXPERIENCE ON ITS BEE CURTAIN DURING THREE DIMENSIONAL DEFLECTIONS BEFORE, DURING AND AFTER SHIMMERING WAVE
}

\author{
Dominique Waddoup and Madhusudan Man Singh* \\ Institute of Zoology, University of Graz, Australia \\ *Research Centre for Applied Science and Technology \\ Tribhuvan University, Kirtupur, Kathmandu \\ E-mail: waddoup@edu.univ-graz.at
}

\begin{abstract}
Giant honeybees (Apis dorsata F.) build open nests far off the ground. As a consequence of their open nesting behaviour giant honeybees seem to be an easy target for predators, such as wasps or birds. However, they have evolved several strategies for defence. Shimmering waves are a defence behaviour, which keep predators far off, by showing visual cues. In the occurrence of shimmering, thousands of colony members, predominantly curtain bees, flip their abdomens in a fraction of a second up to 90 degrees in a rhythmic wave. This effect is comparable to human Mexican waves in the football stadiums. This study gives new insights about how individual bees experience during three dimensional deflections on its bee curtain, before during and after a shimmering wave and how they might decide to participate.
\end{abstract}

Key words: Biphasic movements, focus bees, shimmering, thrusting behaviour, thoraxic motions, z-dimensional movement.

\section{INTRODUCTION}

The giant honeybee Apis dorsata F. is native to southern and south-eastern Asia. This evolutionary older form of the bee genus (Apis) shows open nesting behaviour. Giant honeybees build their single huge comb nests in exposed places far off the ground, such as on tree branches, rocks or even buildings (Koeniger and Koeniger 1980). A giant honeybee nest contains approximately 100.000 individuals and honey stores of $50 \mathrm{~kg}$ and more. It includes a central comb with honey and pollen storages in addition to its reproductive cells. The comb is covered by colony members in multiple layers, which are loosely attached to it. This appearance is termed as the 'bee curtain'. In the 'mouth' zone of the bee curtain, bees arrive and depart and show dancing behaviour. With the exception of this region, curtain bees are quiescent (Kastberger et al. 2010). The upper layer bees remain in a vertical position, with their heads up and connected to their neighbours by their legs (Butler 1962). Due to their position, the curtain bees can be compared with a set of twines hanging down from a solid structure (Weihmann et al. 2009). As a consequence of their open nesting behaviour, giant honeybees appear to be an easy target for predators, but they have developed many strategies for defense. Generally defense behaviours of giant honeybees can be 
divided into physical and non-physical contact with aggressors. Stinging is a very expensive form of defense, as the bee dies afterwards. However, giant honeybees have found an effective solution to keep predators far off them, without coming into physical contact. Therefore, giant honeybees try to repel predators visually by showing shimmering Waves. In the event of Shimmering thousands of colony members, predominantly curtain bees, flip their abdomens in a fraction of a second up to 90 degrees in a rhythmic wave. This effect can be compared to Mexican waves in human aggregations (Farkas et al. 2002) and it is mainly evoked by visual cues (Kastberger, et al. 2010). Abdominal shaking waves can originate from one or more points and they either hiss through the nest from only one direction to the other, or they spread in circles (Kastberger, et al. 2010). Shimmering occurs repetitively and the patterns of waving display and the velocity levels vary. At high predation, the intensity increases and decreases at low intensity. It has been proved that abdominal shaking reduces the risk of predatory wasps significantly, as they react and confused when they are near the nest (Kastberger et al. 2008). During this study, individual bees were identified via stereo tracking. The aims of this study were firstly to investigate if certain bees participate actively or passively in shimmering and further to detect if there are passive deflections during the waving regarding the three dimensions of space and to ascertain differences between the stages of participation. The final aim was to detect the consequences of the geometrical arrangement of the mesh of surface bees during waving.

\section{MATERIALS AND METHODS}

\section{Species and study site}

The research was conducted on the nest of giant honey bee (Apis dorsata F.) at the village of Sauraha at the border of the Chitawan National Park of Nepal from 25-10-2010 to 01-12-2010. The Chitwan district has an area of $2218 \mathrm{~km}^{2}$ and is the main Tarai belt of Nepal with capital town of
Bharatpur. It's lower part has an elevation of $244 \mathrm{~m}$ and belongs to the sub-tropical climate zone. A nest of giant honey bee (Apis dorsata F.) was chosen (Fig. 1) which enabled a practical approach to the bee curtain.

\section{Technical details of study site}

Stimulation by dummy wasp: A dummy wasp was custom-built out of Styrofoam with black and white patterns (Fig. 1c). The replicate was attached to a cable car device, which enabled movement of the dummy automatically in different directions (approaching the nest and reversing) and to variegate velocities. Due to this construction we were able to elicit shimmering waves in roughly controlled directions and intensities.

Video recording: Two stereo cameras recorded $15 \mathrm{~s}$ sessions of behaviours with up to two events of shimmering. The stereo cameras were fixed symmetrically, but in different angles, to a solid carrier and placed in front of the nest (Fig. $1 \mathrm{a}, \mathrm{b}$ ) with a working distance of more than $2 \mathrm{~m}$ and a baseline of $1 \mathrm{~m}$. The stereo systems were able to capture images at a frame rate of 60 frames per second (fps). An industrial PC which contained two frame grabber cards was used to connect the images via camera link. To synchronize both stereo cameras they were interconnected in a master-slave system (Maurer 2010). With this technique the frame grabber card of one camera acts as the "master" of the other and generates a trigger signal that externally triggers the second frame grabber card. Due to the quantity of data, the images were buffered to RAM and later written to disc. This stereoscopic system provided a stable tracking performance, which resolved ambiguities. Thereby it empowered measurement of 3D locations of hundreds of bees densely packed at the surface of a nest. In addition to the stereoscopic system, a high definition camera (Panasonic D2C Pro HD) was used to record the entire experiment. 
Vibrometer: Further, a laser Doppler vibrometer (Polytec PDV 100) additionally detected zdimensional movements (movement towards and away from the nest) of single bees. This scientific instrument directs a laser beam at the surface of interest. Vibration and frequency are extracted from the Doppler shift of the laser beam frequency, due to the motion of the surface. In other words, the laser Doppler vibrometer does not measure distance changes, but velocity differences.

\section{Evaluation of sample waves}

Data selection: On the surface of a giant honeybee nest there are two different regions to distinguish, the quiescent zone and the mouth zone. In the mouth zone, surface bees show less shimmering behaviour. This section of the nest is mainly used for arrival and departure, including dancing behaviours. In contrast, surface bees of the quiescent zone are more likely to demonstrate shimmering behaviour (Kastberger et al. 2010). For this reason a specific area of $700 \times 500 \times 150$ $\mathrm{mm}^{3}(\mathrm{x}, \mathrm{y}, \mathrm{z})$ was chosen in the quiescent region. A sequence of 110 frames which included two singular wave processes was evaluated and focused on 111 surface bees. A frame rate of $60 \mathrm{~Hz}$ with an intercept of $16.67 \mathrm{~ms}$ between two successive frames was chosen. Both waves were triggered by dummy wasp and spread from the right to the left side of the experimental nest.

\section{Stereo tracking}

Identifying individual bees: Synchronous images of a scene recorded with both stereo cameras from different viewpoints were used to identify the surface bees via their position and orientation. This 'segmentation' was the basis for the matching process. The data of both stereo images were combined via normalized cross-correlation and assigned. Consecutively the matched bees were re- identified in video frames (tracking procedure) and triangulated in real-world coordinates. The triangulation which provided geometric information of the bees was retrieved by triangulating the $3 \mathrm{D}$ coordinates of the centre point of the bee thoraces. Therefore, the stereoscopic system was calibrated using the standard calibration method (Maurer 2010). The calibration was repeated once a day to maintain the geometric consistency under field conditions.

Tracking: Two programmes (A, B) written in Java were used to detect movements of all reviewed bees during shimmering in three dimensions of space and distance differences of neighbouring bees to focus bees. Programme A produced a map of the exact location of a defined reference bee to its neighbouring bees. Programme B created a film clip of the reference bee and its neighbours. This empowered us to assign via manual decision, when, and at what intensity the individual bee behaved during shimmering.

\section{Detection of positional deflections of the thoraxes of bees}

To ascertain the status of contribution of a bee in shimmering, programme B placed a tracker on the thorax of the bee, which enabled measurement of the directional components of the motions in 3D (Fig. 3). X-dimensions where measured by sideway movements. Positive values mean a deflection to the right and negative values to the left. The ycomponent shows up and downward directions. Positive ordinate values indicate upward movements and negative values downward. Movement towards and away from the nest is shown by the z-component. Positive values show movement away from the nest (towards the stereo cameras) and negative values show movement towards the bee comb. The measured volume amounts 700x500x150 $\mathrm{mm}^{3}(\mathrm{x}, \mathrm{y}, \mathrm{z})$. 
Defining strength of individual thrusting behaviour

The movement of the bee was manually categorized in one of three stages. The individual bee showed a strong response if the abdomen was lifted more than $45^{\circ}$, when lifted under $45^{\circ}$ it was considered as a medium response. If the individual lifted its abdomen less than $20^{\circ}$, it was regarded as zero abdominal shaking. The first two categories were considered as active and the last as passive.

Defining time zero: Time zero (T0) of the waving appearance was defined as one frame before the bee starts moving. When the bee remained passive, an adjacent bee which actively responded to shimmering was taken as a reference to assign the onset of the wave at the site of the agent bee. Preferably neighbours directly above or beneath the bee had been selected as the wave arrives at them at the same time (Fig. 2b).

\section{Defining neighbourhood between bees}

The bees were chosen manually as shown in Fig. 2c. Bees termed as focus bees are individual bees, which are taken as references to their neighbouring bees. Bees in absolute nearness, or with only a minimal distance (x: $7.3 \mathrm{~mm}, \mathrm{y}: 3.4 \mathrm{~mm}$ ) to the focus bee, were considered as neighbour bees. This means that a bee can only be chosen as a neighbour bee, if there is no other individual between it and the focus bee. One focus bee can have a maximum of 8 neighbour bees.

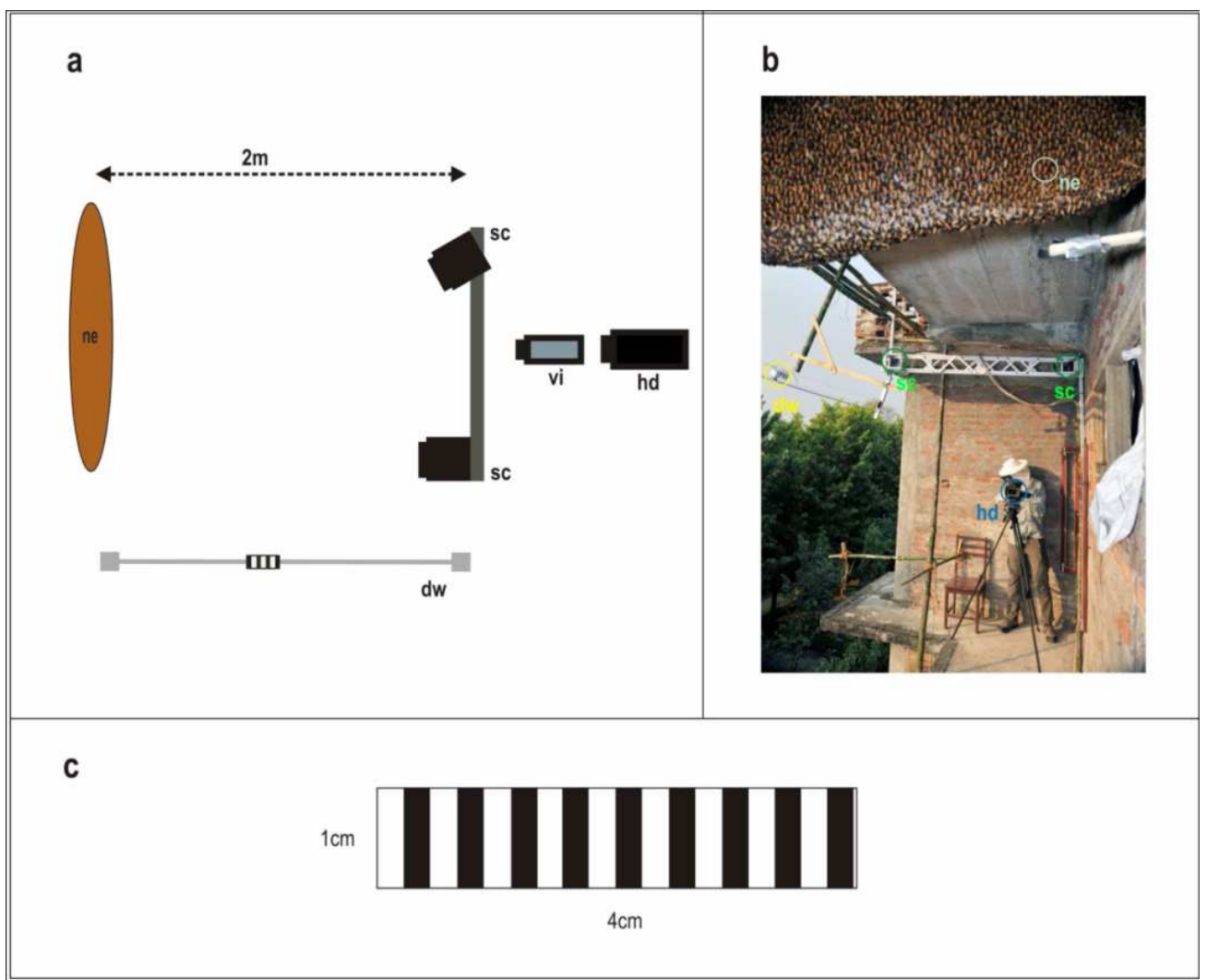

Fig. 1. Experimental setup. a) Schematic view from above. ne: Nest, dw: Cablecar device with dummy wasp, sc: Stereo cameras, vi: Vibrometer, hd: High-Definition Camera; b) Picture of experimental setup on a balcony of a house in Chitwan, Nepal. ne: nest, dw: Cablecar device with dummy wasp, sc: Stereo cameras, hd: High-Definition Camera; c) Dummy wasp with black and white stripes. 


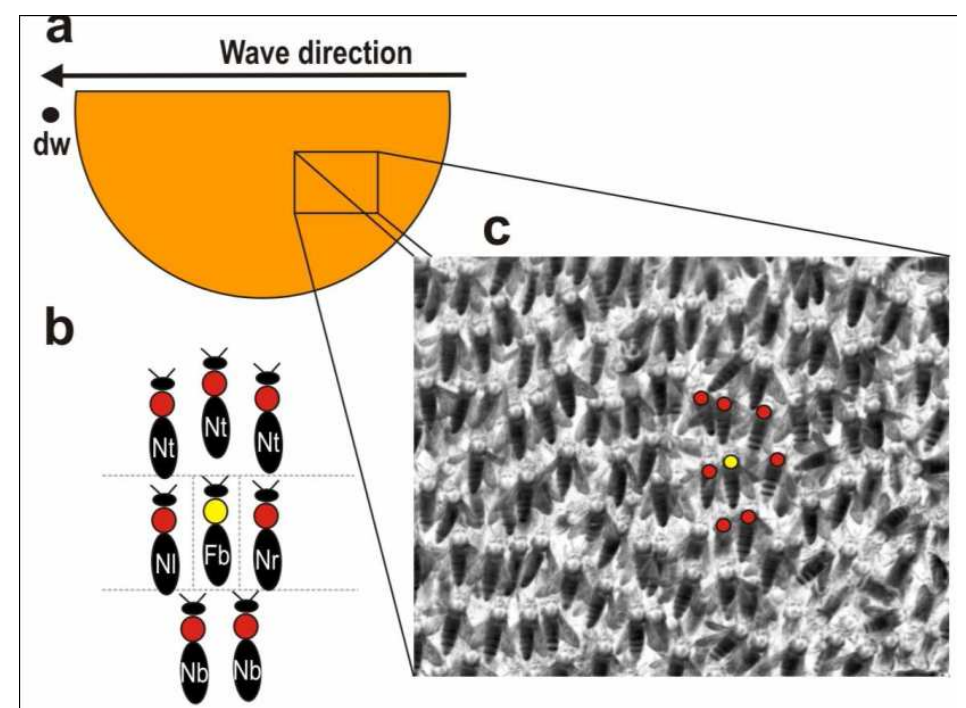

Fig. 2. a) Nest with investigated area measuring $5 \mathrm{~cm} \times 7 \mathrm{~cm}$. Arrow shows the wave direction evoked by the dummy wasp. dw: dummy wasp; b) Sketch of focus bee and neighbouring bees. Fb: Focus bee, Nt: Neighbour bee top, Nr: Neighbour bee right, Nb: Neighbour bee bottom, Nl: Neighbour bee left; yellow circle shows the thorax of the focus bee, red circles show the thoraces of the neighbouring bees. c) Photo of stereo camera. Yellow circle indicates the thorax of the focus bee, red circles show the thoraces of the neighbour bees.

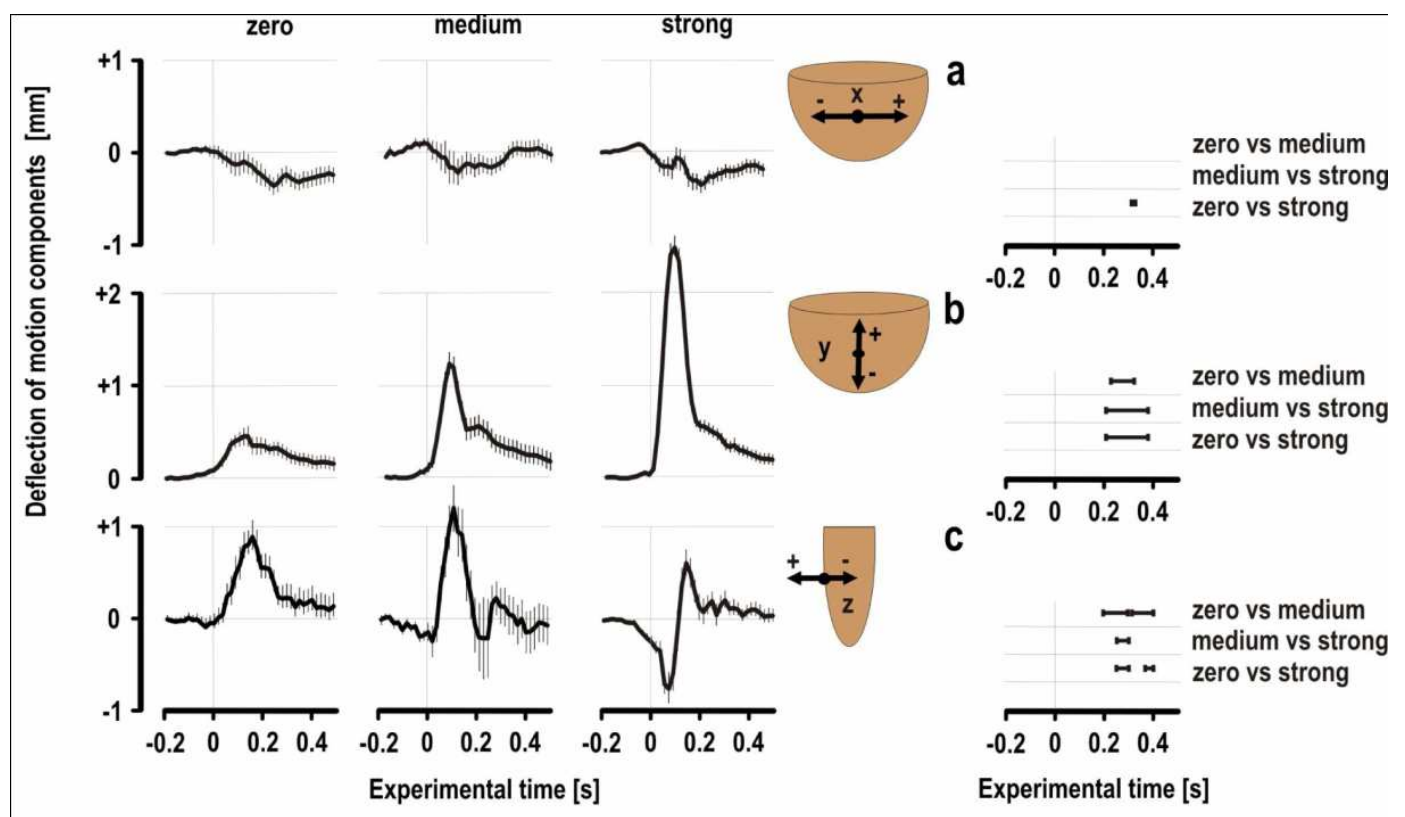

Fig. 3. Shimmering deflections of all bees. a) $x$-axis (right - left movements); b) y-axis (up and down movements); c) z-axis (movements towards and away from the nest). Zero, medium and strong represents the abdominal shaking intensities. When the abdomen is lifted beneath $20^{\circ}$ it is classified as zero abdominal shaking, if lifted up to $45^{\circ}$ it is considered as medium waving participation and when lifted above $45^{\circ}$ it is categorized as strong abdominal shaking. Curves show arithmetical means, thin vertical lines denote mean error. Circles in icons show zero value. Markings in diagrams on the right illustrate statistical significance between the stages of participation $(\mathrm{P}<0.001,2$ Way ANOVA). 


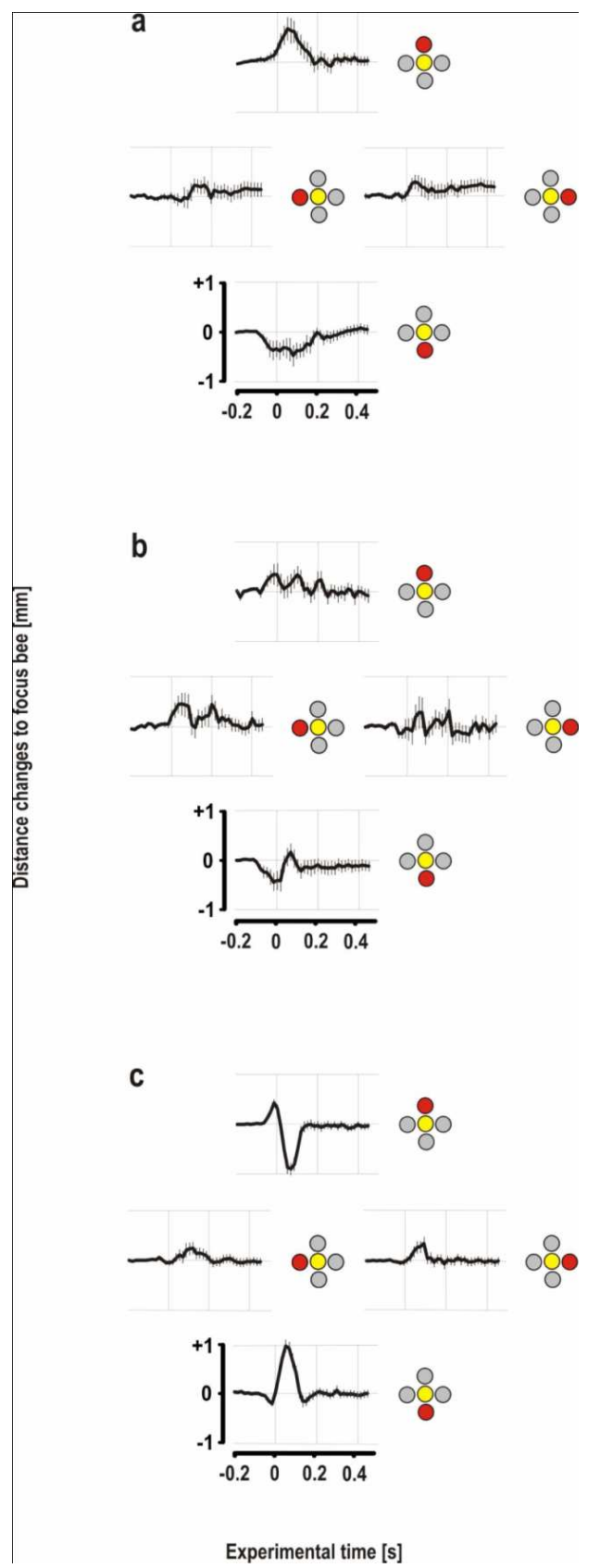

Fig. 4. Distance changes of neighbouring bees to their focus bees during shimmering processes. a) Zero abdominal shaking lifting the abdomen beneath $20^{\circ}$; b) medium abdominal shaking - lifting the abdomen up to $45^{\circ}$; c) strong abdominal shaking - lifting the abdomen over $45^{\circ}$; Curves illustrate arithmetical means, thin vertical lines show mean error. Positive values mean increased distance to the focus bee and negative less distance. The icon next to every diagram shows the position of the neighbour bee and the focus bee. Yellow circle indicates the thorax of the focus bee and the red circle stands for the thorax of a neighbouring bee, whose diagram is showed. The grey circle denotes the other neighbour bees which are not represented in that particular diagram.

\section{Distance changes of neighbouring bees to a focus bee}

It is reviewed with the same method as the single bees, the distance changes of the neighbours to the focus bee. The distance changes before and during the shimmering wave were of special interest (Fig. 4).

\section{Statistics}

For statistical analysis data were assorted via a macro and evaluated with SigmaPlot. Two way ANOVA was used to compare relations.

\section{RESULTS}

\section{Thoracic motions of bees during shimmering}

The abdominal waving strength was categorized manually in three stages of participation and subsequently, 3D deflections were detected via stereo tracking. Positional changes regarding the $\mathrm{x}$-axis do not differ between the stages of participation, which shows that the $\mathrm{x}$ component is mainly a passive effect. In contrast, the y-component clearly shows differences between the abdominal shaking categories $(\mathrm{P}<$ $0.001 ; 2$ Way ANOVA). Likewise all stages of participation differ strongly on the $\mathrm{z}$-axis ( $\mathrm{P}<$ 0.001; 2 Way ANOVA), especially with its biphasicity at strong intensity. Fig. 3 shows that biphasicity is gradually affected by the intensity of shimmering. The z-component shows at all intensities, a minor movement towards the nest before the real shimmering event occurs. The yand the z-values can be regarded as active effects, as they mainly differ between all stages of participation.

\section{Distance changes of neighbours to focus bees during shimmering}

The neighbouring bees were manually chosen (Fig. 2c) and categorized in top, right, bottom and left orientation to the focus bee (Fig. 2b). Positive values indicate increased distance to the focus bee and negative values less. At strong intensity top orientated neighbour bees show biphasic movement, this means they first increase their distance to the focus bee and afterwards decrease their space during a shimmering event (Fig. 4). 
Bottom orientated neighbouring bees only show a slight biphasicity. The neighbour bees at the right side of the focus bee and the neighbours at the left side behave quite similar except that the bees on the right side start slightly earlier with abdominal waving than the bees on the left side. This reaction indicates the direction of the shimmering wave.

\section{DISCUSSION}

This study gives new insights of how single bee experience during three dimensional deflections on its bee curtain before, during and after a shimmering wave and how they might decide to participate. As shown in Fig. 3, the x-axis rarely identifies differences between the stages of participation in it, therefore it can be proposed as a predominantly passive effect. In contrast, the ycomponent is strikingly dependent from active participation during the waving event. Comparably the $\mathrm{z}$-component is also dependent on active participation. Every intensity of the z-signal shows a slight movement towards the nest, before the actual waving event arrives. The bees cling to the subsurface curtain bees with all their six legs. As a result, due to this functional architecture of the bee curtain, the individual bee is able to detect small deflections in the curtain matrix, before the waving occurrence. In this short span of time, the individual bee decides how it participates in shimmering. This is giving evidence that the $\mathrm{z}$ component plays a role for intrinsic communication (Kastberger et al. 2011). Hence, the comparability to human Mexican waves in football stadiums is only limited, as the ground is solid and not moving (Kastberger et al. 2011). Further, the waving information in human produced waves is only passed on visually. Supplementary distance relations between focus bees and their neighbours during shimmering were reviewed. The directions of the waves were viewable, due to slightly earlier distance changes of neighbours situated on the right side of focus bees in comparison to neighbours on the left side. For further research it would be interesting to investigate how neighbour bees behave in comparison to focus bees in the three dimensions of space and in angle relations.

\section{ACKNOWLEDGEMENT}

Authors are thankful to Prof. Dr. Gerald Kastberger, Institute of Zoology, Karl-FranzensUniversitat, Graz, Austria for providing guidance, and encouragement throughout the study period in the research site.

\section{REFERENCES}

Butler, C.G. 1962. The World of The Honeybee. Collins, London.

Farkas, I., D. Helbing and T. Vicsek 2002. Social behaviour: Mexican waves in an excitable medium. Nature 419:131-132.

Kastberger, G., E. Schmelzer, I. and Kranner. 2008. Social waves in Giant honeybees repel hornets. PLoS ONE 3(9):e3141. Doi:10.1371/ journal.pone.0003141.

Kastberger, G., F. Weihmann and T. Hoetzl. 2010. Self-assembly processes in honeybees: The phenomenon of shimmering in honeybees of Asia. Hepburn HR, Randall SE; Chapter 18.

Kastberger, G., F. Weihmann., T. Hoetzl and M. Maurer. 2011. Stereoscopic motion analysis in densely packed clusters demonstrated for the shimmering behaviour in Giant honeybees. Frontiers of Zoology 8:3. http://www.frontiers inzoology.comcontent/8/1/3.

Koeniger N. and G. Koeniger. 1980. Observations and experiments on migration and dance communication of Apis dorsata in Sri Lanka. $J$ Apic Res. 19(1):21-34.

Maurer, M. 2010. Rigid body reconstruction for motion analysis of giant honeybees using stereo vision. Master's Thesis. Institute for Computer Graphics Vision (ICG) Graz University of Technology, Inffeldgasse 16, 6010 Graz, Austria.

Weihmann, F., T. Hoetzl and G. Kastberger. 2009. 3-D patterning of social waves in the Giant honeybee Apis dorsata. Proceedings of the Deutsche Zoologische Gesellschaft (Regensburg). 